\title{
Directional shear jamming of frictionless ellipses
}

\author{
Martin Trulsson (1) ${ }^{*}$ \\ Theoretical Chemistry, Lund University, Lund SE-221 00, Sweden
}

(Received 9 February 2021; accepted 22 September 2021; published 28 October 2021)

\begin{abstract}
In this work we study shear reversals of dense non-Brownian suspensions composed of cohesionless elliptical particles. By numerical simulations, we show that a new fragility appears for frictionless ellipses in the flowing states, where particles can flow indefinitely in one direction at applied shear stresses but shear jam in the other direction upon shear stress reversal. This new fragility, absent in the isotropic particle case, is linked to the directional order of the elongated particles at steady shear and its reorientation at shear stress reversal, which forces the suspensions to pass through a more disordered state with an increased number of contacts in which it might get arrested.
\end{abstract}

DOI: 10.1103/PhysRevE.104.044614

\section{INTRODUCTION}

Both granular matter and dense suspensions have been studied extensively during the last decades due to their industrial and geological relevance and rich physics [1]. The rheology of these particle packings under various circumstances is currently an especially hot topic, with many questions unresolved, including the exact divergence of the viscosity close to shear jamming and the associated universality class [2,3] and how to formulate a statistical toolbox for zero-temperature and amorphous states [4] analogous to the statistical thermodynamic toolbox for equilibrium systems. Even the simplest case of a suspension composed of only repulsive particles at zero temperature shows a vibrant flora of rheological phenomena [5,6], e.g., transient shear banding [7] and discontinuous shear thickening [8-11].

One remarkable property of suspensions is that even though the particles flow in a Stokes flow, particles do not always respect reversibility when exposed to shear reversal [12,13], a property usually associated with Stokes flows [14]. These irreversible particle trajectories, seen in suspensions after strains typically of the order of unity after a shear reversal, lead to a diffusive motion of particles in oscillatory shear flows with high strain amplitudes. For lower oscillatory strain amplitudes, particles land in self-absorbed states [13], showing perfect reversibility. Naturally, there exists a density-dependent critical strain that delimits the reversibleto-irreversible dynamics $[15,16]$. The effect is linked to the collisions between the suspended particles, which distort the particles' trajectories from the fluid streamlines. The self-absorbed states correspond to states where the particles

*martin.trulsson@teokem.lu.se

Published by the American Physical Society under the terms of the Creative Commons Attribution 4.0 International license. Further distribution of this work must maintain attribution to the author(s) and the published article's title, journal citation, and DOI. Funded by Bibsam. restructure themselves to avoid further collisions and hence follow the fluid's streamlines, i.e., show reversibility. While first discovered for semidilute suspensions, this mechanism has recently been rediscovered for dense suspensions [17-19], leading to dethickened states at low oscillatory shear strains. These dethickened states are naturally linked to self-absorbing states, as both correspond to zero or very few particle collisions. Small oscillatory strains seem to deactivate frictional forces, leading to higher shear-jamming packing fractions for frictional particles [19]. The deactivation of frictional forces is perhaps more evident when one performs shear reversal experiments on dense suspensions [17], where it takes a finite strain, typically around 1, before the suspension has restructured and activated the frictional contacts again. Shear-jammed suspensions also show a finite strain over which the suspensions are flowable in the reverse direction [20], illustrating the fragility of these shear-jammed configurations. This critical strain varies with the packing fraction, starting at around unity close to the shear-jamming packing fraction and decreasing as the packing fraction increases. Even though fragility has been explored in the jammed region, it has never been seen in the flowing state, i.e., below shear jamming. Here we show that a new kind of fragility appears for suspensions composed of elliptical particles below the steady shear jamming. This fragility is a new reversible-irreversible transition acting on a suspension as a whole, where a suspension flow becomes irreversible in the sense that it flows indefinitely in one shear direction but shear jams in the opposite direction, i.e., is mechanically stable in one direction but not the other. This new fragility is different compared to both the fragility studied in Refs. [21] and [22], where the authors studied frictional particles starting from stress-free samples and above the corresponding systems yield stress, as well as the undirected reversible-irreversible transition of the dynamics of isotropic particles [12] in oscillating shear flows.

\section{MODEL}

We carry out extensive numerical simulations of nonBrownian suspensions composed of an amorphous ensemble 
of frictionless elliptical particles (in two dimensions) in planar shear flows. We study ellipses with various aspect ratios $\alpha$, defined as the ratio between the length of the major $a$ and that of the minor $b$ axis, from 1 (disks) to 3 and at packing fractions up to their respective shear-jammed states. The particles interact via simple harmonic potentials, where the force is proportional to the overlap as $\mathbf{f}_{i j}=k \boldsymbol{\delta}_{i j}^{\perp}$, where $k$ is the spring constant and $\delta_{i j}^{\perp}$ the overlap, normal to the surface, between the two ellipses $i$ and $j$. Since the ellipses are anisotropic and force is normal to the surface, the ellipses are also subjected to torques in relation to the forces. For more information about the contact forces, see [23]. The amorphous ensemble is generated by choosing the major axis length of an ellipse randomly from a flat distribution between $a /\langle a\rangle=[0.5,1.5]$, all with the same aspect ratio. Particles are quasihard, where the ratio $k /\langle P\rangle$ is set so high (typically above $10^{3}$ ) that the obtained results are insensitive to the value of $k$ (within the noise of the data) and where $\langle P\rangle$ is the average steady shear pressure (normal stress on the walls).

The fluid is described as a continuum with a viscosity $\eta_{f}$. We impose a linear shear profile with the fluid velocity in the $x$ direction equal to $u_{f, x}(y)=\dot{\gamma} y$, where $\dot{\gamma}$ is the shear rate. The fluid velocity field respects nonslip conditions in the vicinity of the walls, i.e., $\dot{\gamma}=\Delta u_{w, x} / H$, where $\Delta u_{w, x}$ is the lateral velocity difference and $H$ the separation between the two walls. This fluid gives rise to viscous drag forces and torques on the suspended particles, the latter giving rise to Jeffrey orbits where the particle angular velocity depends on the orientation. The viscous drag and torque on a ellipse are given by [24]

$$
\mathbf{f}_{i}^{\mathrm{visc}}=-3 \pi \eta_{f}\left[c_{f a}\left(\mathbf{u}_{i}^{a}-\mathbf{u}_{f}^{a}\left(y_{i}\right)\right)+c_{f b}\left(\mathbf{u}_{i}^{b}-\mathbf{u}_{f}^{b}\left(y_{i}\right)\right)\right]
$$

and

$$
\tau_{i}^{\mathrm{visc}}=4 \pi \eta_{f} a_{i} b_{i}\left[\left(c_{M a}\left(e_{i, x}\right)^{2}+c_{M b}\left(e_{i, y}\right)^{2}\right) \Omega-c_{M r} \omega_{i}\right],
$$

where $e=\sqrt{1-\alpha^{-2}}$ is the eccentricity, $\eta_{f}$ is the interstitial fluid viscosity, $\mathbf{u}_{i}^{(a / b)}$ are the translational velocities along the major and minor axes, respectively, of particle $i, \mathbf{e}_{i}=$ $\left(e_{i, x}, e_{i, y}\right)$ is the unit direction vector of particle $i$ 's major axis, $\Omega=\frac{\dot{\gamma}}{2}$ the vorticity of the fluid, $\omega_{i}$ the angular velocity of particle $i$, and $c$ are constants [24]:

$$
\begin{aligned}
c_{f a} & =\frac{8}{3} e^{3}\left[-2 e+\left(1+e^{2}\right) \log \left(\frac{1+e}{1-e}\right)\right]^{-1}, \\
c_{f b} & =\frac{16}{3} e^{3}\left[2 e+\left(3 e^{2}-1\right) \log \left(\frac{1+e}{1-e}\right)\right]^{-1}, \\
c_{M a} & =c_{f a} \\
c_{M b} & =\left(1-e^{2}\right)^{-1} c_{f a} \\
c_{M r} & =\frac{4 e^{3}\left(2-e^{2}\right)}{3\left(1-e^{2}\right)}\left[-2 e+\left(1+e^{2}\right) \log \left(\frac{1+e}{1-e}\right)\right]^{-1} .
\end{aligned}
$$

Particle dynamics is overdamped with force and torque balances on each particle. The effect of adding lubrication forces and details of the then modified model are provided in Appendix B.

We shear $\sim 900$ particles, all having the same aspect ratio $\alpha$ but various sizes, between two rough walls constructed from the same kind of particles as the flowing particles, randomly oriented (the effects of wall roughness and finite size are provided in Appendixes $\mathrm{C}$ and $\mathrm{D}$ ). These walls are rough enough to avoid particle slip. We shear the suspensions at fixed packing fractions by applying shear stress on the two walls, with a stress difference denoted $\sigma$ (equivalent to a constant force difference on the walls), which are sheared for roughly 10 strains, sufficiently long enough to reach steady state, before we do a shear stress reversal (by instantaneously flipping the sign of $\sigma$ ). As the model does not contain any friction, fluid or particle inertia, or soft repulsive or attractive forces (i.e., we are considering a quasi-hard-core model) the rheology is rate independent. Doubling the shear rate will double the shear stress, and vice versa, leading to a rateindependent shear viscosity, i.e., Newtonian behavior. Starting configurations for the constant volume and boundary stress simulations are all prepared by a pressure-controlled rheometer where an external pressure $P_{\text {ext }}$ is imposed at the walls and with a constant $\Delta u_{w, x}$, resulting in an almost-fixed shear rate after a brief period. The desired packing fraction is then obtained by slowly decreasing or increasing the shear rate under the imposed pressure until the desired value is reached, after which we lock the wall separation. Unless specified, we used wall separations of $H / \sqrt{a b} \simeq 20$ and $W / \sqrt{a b} \simeq 48$, where $W$ is the (periodic) box dimension in the $x$ direction. The studied packing fractions correspond to average steadystate viscous numbers $J=\frac{\eta_{f}\langle\dot{\gamma}\rangle}{\langle P\rangle}$ in the range from $3 \times 10^{-4}$ (highest studied packing fraction) to $10^{-2}$ (lowest studied packing fraction), where $\langle\dot{\gamma}\rangle$ is the average steady-state shear rate at the imposed shear stress and fixed packing fraction. Corresponding stress ratios $\sigma /\langle P\rangle$ are typically around $\sim 0.1$. In units of $k$ and $\eta_{f}$, we usually have $\sigma \sim 10^{-4} k$ and $\langle\dot{\gamma}\rangle$ between $\sim 10^{-5} \frac{k}{\eta_{f}}$ (the lowest studied packing fraction) and $\sim 3 \times 10^{-7} \frac{k}{\eta_{f}}$ (the highest studied packing fraction). In total, we sample 10 independent shear reversals per packing fraction $\phi$.

\section{Average direction and nematic order parameter}

The average direction $\theta_{\mathbf{e} \cdot \hat{\mathbf{y}}}$ with respect to the $y$ axis, with the unit vector $\hat{\mathbf{y}}$, is sampled by taking the average orientation of all ellipses in the center of the cell, excluding the five closest layers close to each wall. The nematic order parameter is obtained from the instantaneous director tensor $\mathbf{Q}_{l k}=$ $\frac{1}{N} \sum_{i=1}^{N}\left(2 e_{i, l} e_{i, k}-\delta_{l k}\right)$, where $\delta_{l k}$ is the Kronecker delta and where $l$ and $k$ are either $x$ or $y$. The nematic order parameter is sampled for the $N$ particles in the center of the cell, as done for the average direction. The nematic order parameter is then obtained as $S_{2}=\sqrt{\mathbf{Q}_{x x}^{2}+\mathbf{Q}_{x y}^{2}}$.

\section{RESULTS}

The key finding is reported in Fig. 1, where we report the strain $\gamma$ for 10 independent shear stress reversals at four different packing fractions below the shear-jamming packing fraction for ellipses, estimated to be equal to $\phi_{c}=0.892$ for $\alpha=3$ [23]. For packing fractions considerably below $\phi_{c}$ [see Figs. 1(a) and 1(b)], both the prereversal and the postreversal regions show strains that scale linearly in time with the 


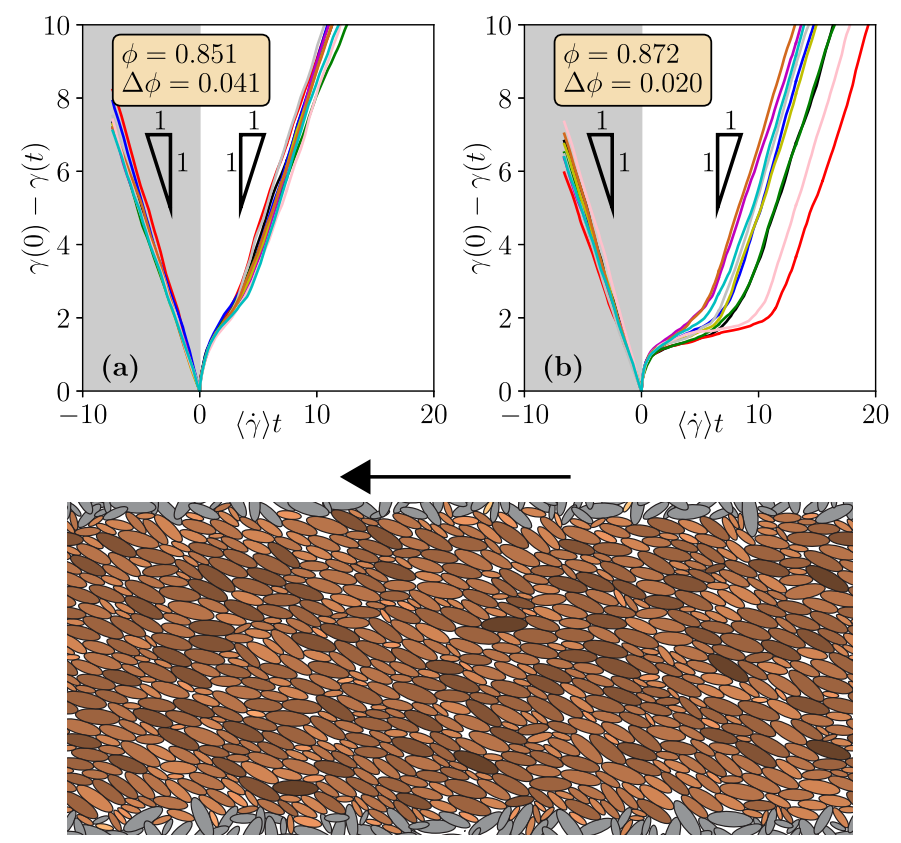

(e)
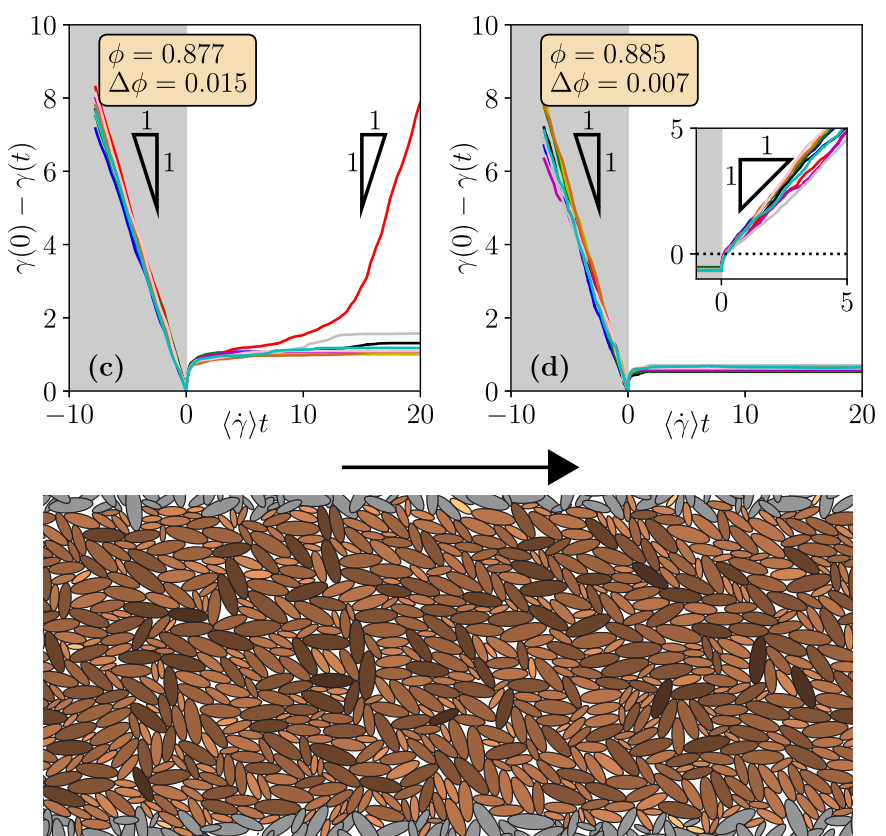

(f)

FIG. 1. Strain $\gamma$ as a function of the rescaled time $\langle\dot{\gamma}\rangle t$ for 10 independent shear-stress reversals (indicated by various colors) for ellipses with $\alpha=3$. The gray-shaded area indicates prereversal evolution, and the white postreversal (upon an instantaneous shear stress reversal, $\sigma \rightarrow-\sigma)$. The effect of increasing the packing fraction is shown, from (a) to (d), with $\Delta \phi=\phi_{c}-\phi$. Proportionality triangles show the slopes -1 (left) and 1 (right), respectively. (e) A typical configuration for a flowing state and (f) that of a shear reversed jammed state at $\phi=0.877$. Arrows indicate the direction of the shear. Gray particles are wall particles, and brown ones flowing particles (the darker, the more contacts). Inset in (d): The strain evolution upon a second shear reversal from the jammed configurations in the figure.

average steady shear rate $\langle\dot{\gamma}\rangle$, as expected for steady flows. After the shear stress reversals, the strains show deviations from a linear scaling, indicating that some relaxation process is involved. Furthermore, this region consists of two regions: one with nonlinear behavior occurring up to strains of $\sim 1$, followed by a quasilinear regime up to a strain of $\sim 2$. In general, the shear rate changes sign almost immediately after shear stress reversal. Increasing the packing fraction further, shear-jammed states start to appear upon shear reversal [see Figs. 1(c) and 1(d)]. For $\phi=0.877,9$ of 10 realizations get arrested in shear-jammed states upon shear reversal after a strain of roughly 1 . For an even higher packing fraction, none of the 10 systems flows in the reverse direction. Even if the packings get arrested in the reverse direction, they immediately start to flow if the stress switches sign a second time [see the inset in Fig. 1(d)]. These flows' strain evolutions, from slow to arrested at increasing density, after a shear reversal from a flowing state, resemble the typical mean-squared displacement curves and their density dependence for hard-sphere glassy systems [25]. The differences compared to a glassy system are that (i) this effect occurs in an athermal system and (ii) this is a macroscopic/collective rheological measurement rather than an averaged one-particle quantity.

Nevertheless, there exist apparent similarities to glassy particle systems, both being arrested systems. Two typical configurations, one of a flowing state and one of an arrested state (upon shear reversal), at $\Delta \phi=\phi_{c}-\phi=0.015$, are depicted in Figs. 1(e) and 1(f). Upon visual inspection, we see a higher directional order characterizing the flowing state than the arrested one.
To better grasp the microscopic origin of these newly found arrested states, and motivated by our above visual inspection, we analyze the systems in terms of directional ordering, both the average direction and the nematic order parameter of the particles. Figure 2 shows the instantaneous angle (in degrees), averaged over the particles in the center of the cell, before and after the shear stress reversal. In the prereversal regime, the particles' average orientation with respect to the $y$ axis $\theta_{\mathbf{e} \cdot \hat{\mathbf{y}}}$ fluctuates around its average steady-state value $\left\langle\theta_{\mathbf{e} \cdot \hat{y}}\right\rangle$, close to $60^{\circ}$ for this particular system. At the lowest reported $\phi$ [see Fig. 2(a)] the direction slowly switches sign upon reversal and saturates to its negated value, $-60^{\circ}$, at around $\langle\dot{\gamma}\rangle t \approx 5$. The saturation in Fig. 2(a) correlates well with the reappearance of linear scaling of the strain with respect to time [see Fig. 1(a)]. Hence, the deviation from linear scaling in the postreversal region can be attributed to the slow reorientation of the direction of the ellipses to the new reversed stress direction. In terms of strain, this completed reorientation corresponds to approximately 2 in the reverse direction, extracted from the data in Fig. 1(a). A single ellipse with $\alpha=3$ in a shear flow has a turnover strain roughly equal to 4.3 . However, a $60^{\circ}$ to $-60^{\circ}$ reorientation corresponds roughly to two strains [see the inset in Fig. 2(a)] and is comparable to what we find in the simulations. This finding is similar to what was found experimentally [26] and numerically [27,28] regardimg anisotropic particles using constant-shear-rate rheometers, even though the relaxation (in terms of strains) seems to depend a bit on the system.

We see similar trends increasing the packing fraction, where the linear scaling and a fully reoriented direction of the 

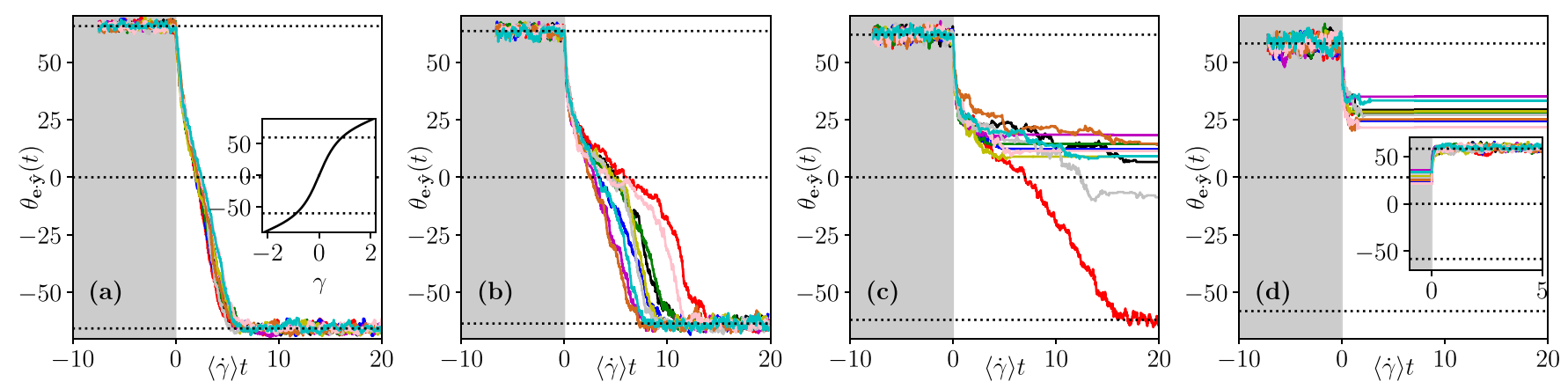

FIG. 2. Average particle direction $\theta_{\mathbf{e} \cdot \hat{\mathbf{y}}}$ (in degrees) with respect to $\hat{\mathbf{y}}$ before (gray-shaded area) and after (white area) shear reversal. Packing fractions (a)-(d) as in Fig. 1. Lines show the average direction of the ellipses $\pm\left\langle\theta_{\mathbf{e} \cdot \hat{\mathbf{y}}}\right\rangle$ in steady shear and the zero line. Inset in (a): The evolution of the angle of a single ellipse in a shear flow as a function of the strain. Doing a turn from $-60^{\circ}$ to $-60^{\circ}$ (indicated by the dashed lines), passing through 0 , requires roughly a strain of 2. Inset in (d): As that in Fig. 1(d), but for the average particle direction evolution.

ellipses coincide. This observation is valid for all the cases where we find a flowing state in the reverse direction. For the arrested states, most configurations get stuck with an average angle of the same sign as for the prereversal shear direction.

Further insights can be obtained if one also looks at the instantaneous nematic order $S_{2}$, as shown in Fig. 3. Following previous results [26-28], the nematic ordering starts to decrease after a reversal until it reaches a minimum, with a corresponding value of approximately 0 for $\theta_{\mathbf{e} \cdot \hat{\mathbf{y}}}$, after which it increases back to its original value. For the studied cases, we see a nematic order parameter $S_{2}$ above 0.9 (high nematic order) at steady shear but as low as 0.2 (low nematic order) after a shear stress reversal. Hence, to reorient the flow in the reverse direction, one passes through a more disordered state (in terms of nematic ordering). While for $\phi=0.877$, the system gets jammed in this disordered state [see Fig. 1(f)], with low nematic ordering and no strong preferential direction along with the flow, this is not the case at even higher packing fractions, where the system instead gets stuck in a more ordered state (compared to the $\phi=0.877$ case) with both a moderate-high nematic order, but still less ordered than in steady state, and a clear antialignment compared to the new stress direction. Hence, these arrested states can reach different degrees of nematic disorder and average particle orientations.

We now turn our attention to the pressure evolution upon shear reversal. Figure 4 shows the rescaled instantaneous normal stress on the walls as a function of time at various packing fractions. For the lowest reported packing fraction, we see a small but still significant maximum. This maximum coincides nicely with when the suspension has its minimum in nematic order and $\theta_{\mathbf{e} \cdot \hat{\mathbf{y}}} \approx 0$, i.e., a fairly disordered configuration with no preferential direction in either flow direction. The pressure maximum increases up to roughly 3.5 times the steady-state value at even higher packing fractions. For the highest packing fraction, the pressure saturates to a value lower than this maximum, most likely because the pressure is not fully developed, as the suspension gets arrested before the most disordered state is reached.

This pressure increase can be understood if one considers the average number of contacts per contact $Z$ and its evolution upon shear reversal: see Fig. 5, where the pressure increase correlates well with an increased number of contacts compared to its steady-state value. As the packing fraction increases, so does the average number of contacts per particle, both at steady state and upon shear reversal. At steady state, a suspension with $\alpha=3$ shear jams when $Z=5.63 \pm 0.02$ [23], indicated by dashed red lines in Fig. 5. Directional shear-jammed states appear when $Z$ reaches or exceeds this steady-state number. Hence, upon shear reversal, the elliptical particles pass through a more disordered state with a higher number of contacts, possibly leading to shear jamming. It, furthermore, indicates that the shear jamming is controlled by the number of contacts per particle rather than the packing fraction and that the $Z$ value at which the particles jam is roughly independent of the value of the nematic order parameter.
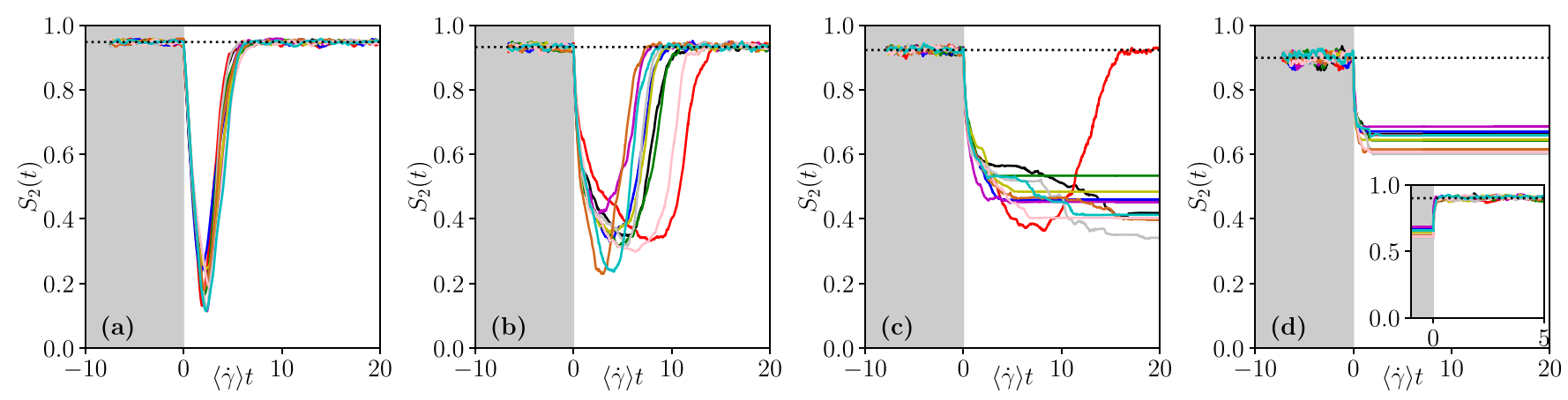

FIG. 3. Nematic order parameter $S_{2}$ before (gray-shaded area) and after (white area) shear reversal. Packing fractions (a)-(d) as in Fig. 1 . Lines show the corresponding averaged value $\left\langle S_{2}\right\rangle$ as obtained at steady state. Inset in (d): As that in Fig. 1(d), but for $S_{2}$. 

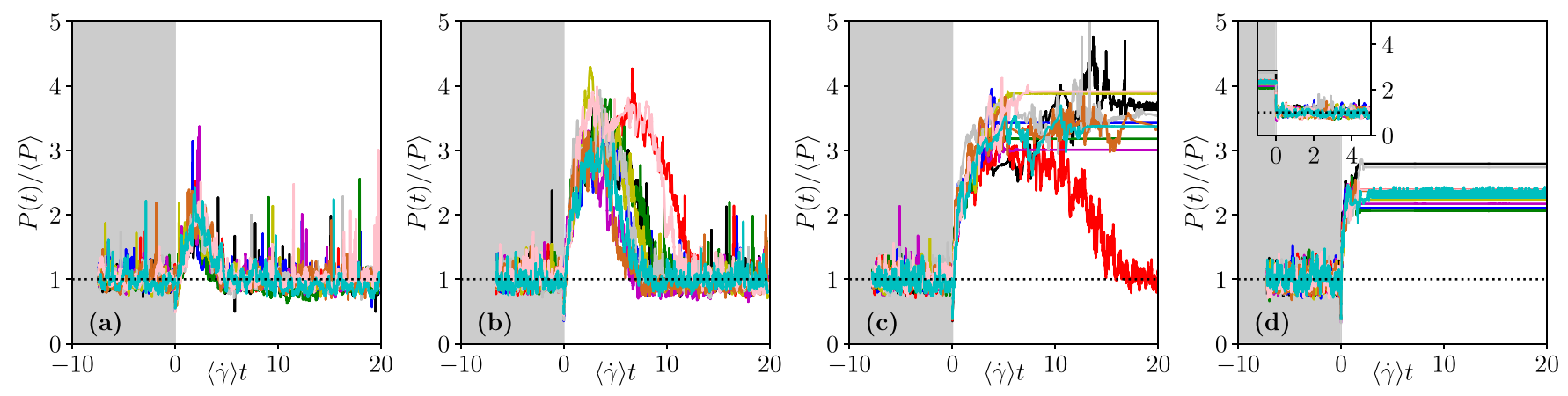

FIG. 4. Instantaneous rescaled pressure $P /\langle P\rangle$ at the two walls as a function of the rescaled time $\langle\dot{\gamma}\rangle t$. Packing fractions (a)-(d) as in Fig. 1 . Dashed lines show the average pressure $\langle P\rangle$ at steady state. Inset in (d): As that in Fig. 1(d), i.e., for a second shear reversal, but with $P /\langle P\rangle$.

After seeing the above results, one natural question arises: At what aspect ratio do these arrested states appear? In order to answer this question, we carry out additional simulations for suspensions composed of particles with lower aspect ratios. It turns out that directional arrested states start to appear as soon as the aspect ratio is greater than roughly 2.2 (see Fig. 6 and Appendix A). The range over which one can observe this directional shear jamming also increases with an increasing aspect ratio. Interestingly, this is also the reported aspect ratio at which monolayered colloidal ellipsoids (two-dimensional systems) start to have two distinct glass transitions densities: a lower one for the rotational motion and a higher one for the translational one [29].

One could imagine that the rough boundary caused this directional shear jamming. Likewise, one could argue that lubrication forces would increase the flowability of the system, i.e., lubricate the system and, hence, eliminate the phenomenon. However, as shown in Appendixes B and C, none of these effects changes the results. Appendix $\mathrm{E}$ also highlights that the chosen spring constant is high enough not to influence the results. Furthermore, the simulated system is large enough to avoid finite-size effects. These results strongly indicate that these effects $[30,31]$ are not the cause of the (directional) shear jamming.

\section{SUMMARY}

In summary, we have presented a new type of fragility present in dense non-Brownian suspensions composed of anisotropic particles. We have illustrated this for elliptical particles with an aspect ratio $\alpha=3$. Even though the suspension behaves as a Newtonian fluid when shear stress is applied in one direction, it shear jams when the stress is applied in the reverse direction for packing fractions greater than $\phi>\left(\phi_{c}^{\alpha=3}-0.015\right)$. For ellipses this fragility, or directional shear jamming, starts to be important for $\alpha>2.2$ and packing fractions close to the steady-state shear jamming. These novel fragile states are fundamentally different from the fragile states seen for isotropic and frictional particles [21], as those systems shear jam in any direction given that the strain is large enough. Furthermore, it is not unlikely that spherocylinders $[28,32]$ are more prone to show this fragility due to their more localized curvature compared to ellipses. Since granular materials and dense non-Brownian suspensions share much physics [33-35], it would not come as a surprise if this fragility also exists for granular materials, possibly shifted due to inertial effects. Investigation of this effect for granular material as well as for frictional particles [23] will thus be a natural extension of this work.

\section{ACKNOWLEDGMENTS}

The author thanks J. Stenhammar for valuable comments. The simulations were performed on resources provided by the Swedish National Infrastructure for Computing (SNIC) at the Center for Scientific and Technical Computing at Lund University (LUNARC).
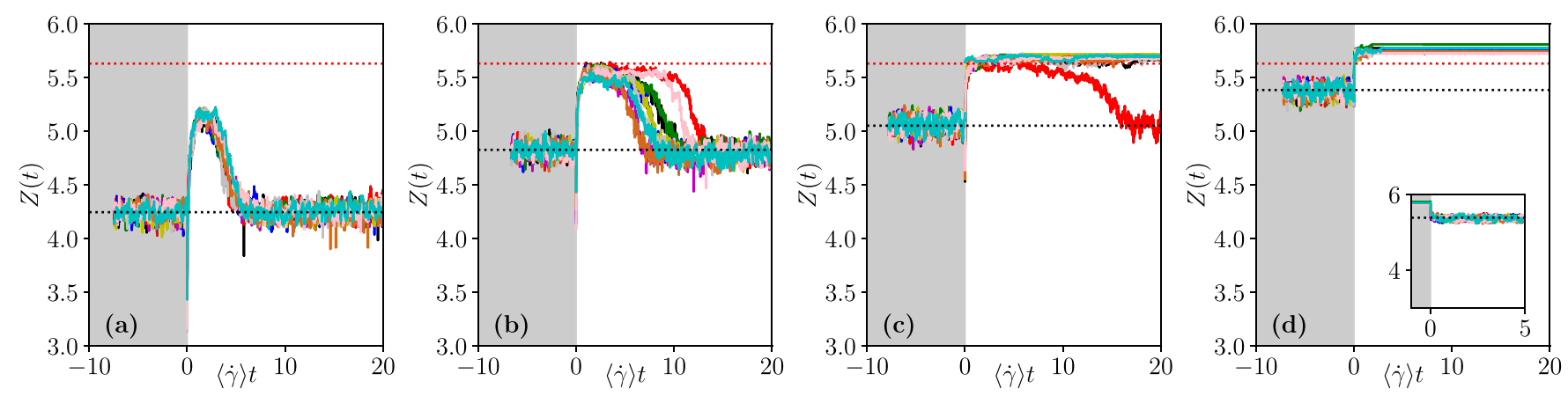

FIG. 5. Instantaneous number of contacts per particle $Z$ as a function of the rescaled time $\langle\dot{\gamma}\rangle t$. Packing fractions (a)-(d) as in Fig. 1 . Dashed black lines show the average values of $\langle Z\rangle$ at steady state. Dashed red lines show the critical value at steady-state shear jamming (value from Ref. [23]). Inset in (d): As that in Fig. 1(d), i.e., for a second shear reversal, but for $Z$. 


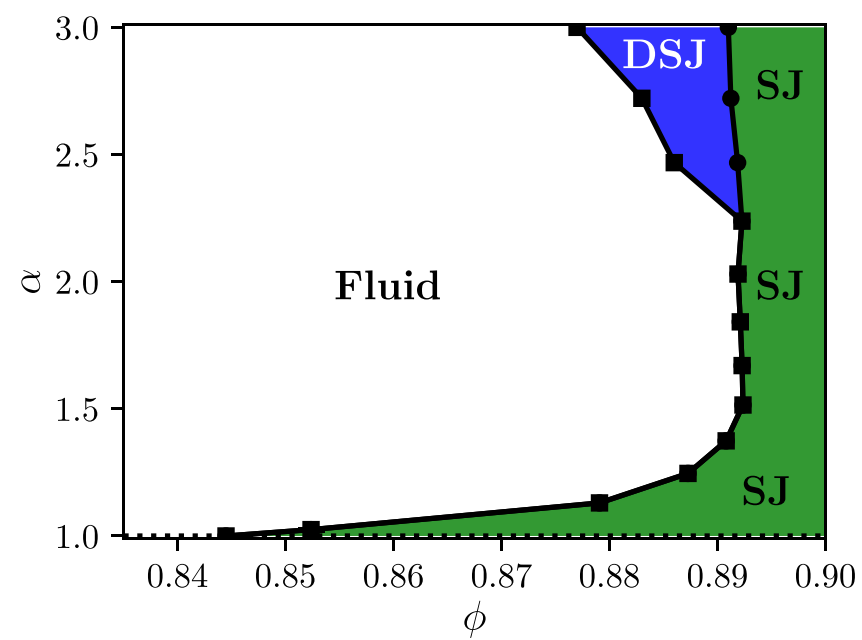

FIG. 6. Jamming phase diagram in the $\alpha-\phi$ plane. The diagram shows the fluid, directional shear-jamming (DSJ), and shearjamming (SJ) regions.

\section{APPENDIX A: SHEAR REVERSAL AT LOWER ASPECT RATIOS}

Figure 7 shows the strain, instantaneous direction angle, nematic order parameter, pressure, and number of contacts per particle evolution for suspensions with aspect ratios $\alpha<3$ at low and comparable relative distances to their respective shear-jamming packing fractions, $\Delta \phi \sim 0.01$. At $\alpha=1$ (disk particles) and $\alpha=2.04$, the linear scaling in the strain as a function of time is almost immediately recovered upon shear reversal without any significant maximum pressure. The directional angle and nematic order parameter for $\alpha=2.04$ also relax almost immediately. For higher aspect ratios, strain and directional angle relaxations become slower (in terms of accumulated strains) with an appearance of intermediate quasilinear regimes with smaller absolute slopes than in steady shear. At a high enough aspect ratio the slope becomes zero and the intermediate regime becomes permanent (i.e., a shear jammed state). These higher aspect ratios also show clear maxima in pressure and the number of contacts after a shear reversal.

\section{APPENDIX B: EFFECT OF LUBRICATION FORCES}

To verify that directional shear jamming is not diminished by short-range lubrication forces, we carry out additional simulations with lubrication forces included. The lubrication is modeled as previously done for disks [36]. The squeeze mode (normal to the surfaces) is given by

$$
\mathbf{f}_{\text {lub }, n}^{i j}=-\frac{3 \pi}{8} \eta_{f} \kappa_{i j}^{-1}\left[\frac{\left(\mathbf{V}_{i}-\mathbf{V}_{i}\right) \cdot \mathbf{n}_{i j}}{h_{i j}^{\perp}+\delta_{\text {rough }}}\right] \mathbf{n}_{i j},
$$

where $\kappa_{i j}^{-1}=\frac{2 \kappa_{i}^{-1} \kappa_{j}^{-1}}{\kappa_{i}^{-1}+\kappa_{j}^{-1}}$ is a reduced inverse curvature using the curvatures, $\kappa_{i}$ and $\kappa_{j}$, of the two ellipses at the closest contact points, $\mathbf{n}_{i j}$ the normal unit vector to the two surfaces, $\mathbf{V}_{\mathbf{i}}$ the total velocities (i.e., including both translational and rotational motions) at the closest contact points of ellipse $i, h_{i j}^{\perp}$ the gap perpendicular to the surface between the two closest points,
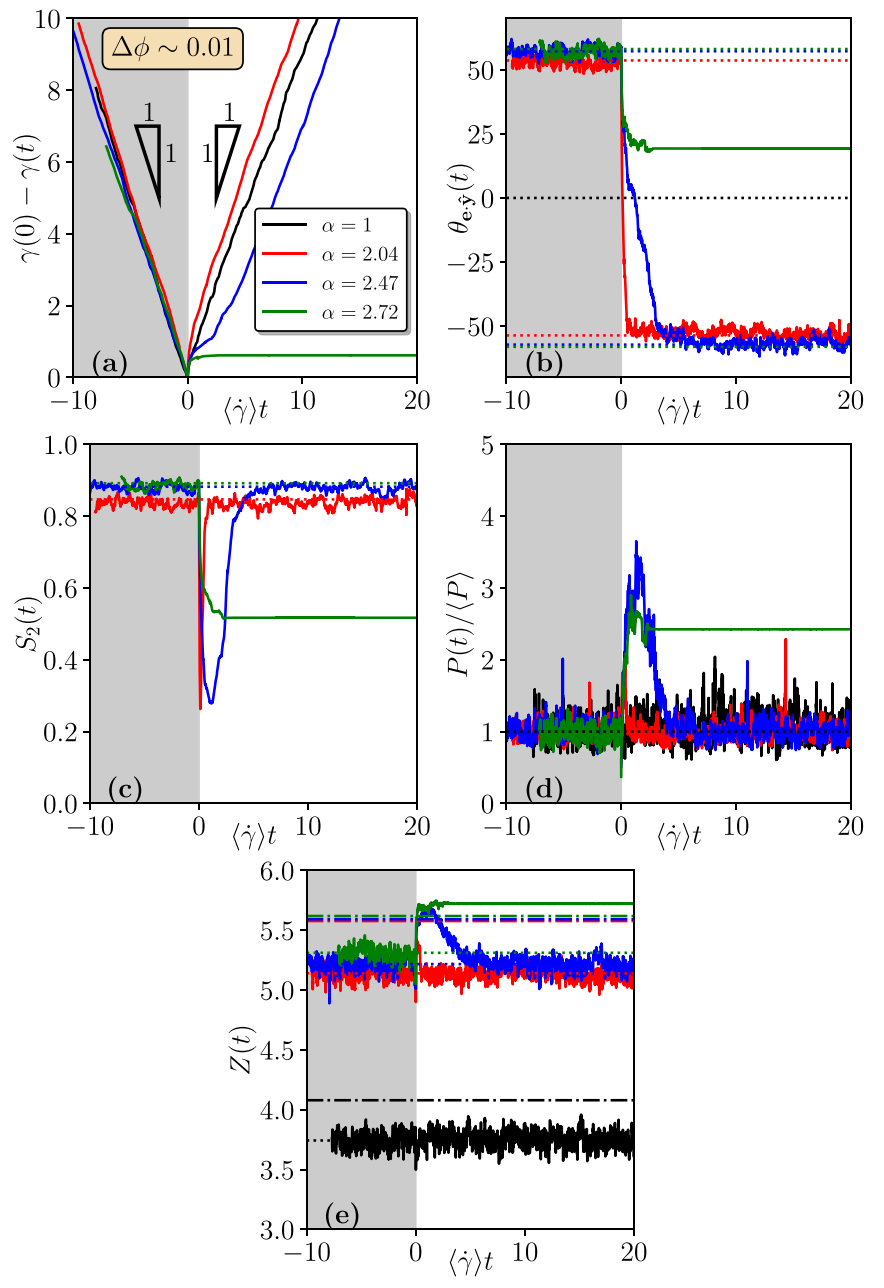

FIG. 7. (a) Strain $\gamma$, (b) average particle direction $\theta_{\mathbf{e} \cdot \hat{\mathbf{y}}}$ (in degrees) with respect to $\hat{\mathbf{y}}$, (c) nematic order parameter $S_{2}$, (d) instantaneous pressure at the two walls, rescaled by its corresponding steady shear value, and (e) average number of contacts per particle as a function of time for four different aspect ratios as indicated by the legend in (a). Dotted lines in (b), (c), and (e) show the corresponding values at steady shear. Dashed-dotted lines in (e) show the corresponding value of $Z$ at steady-state shear jamming.

and $\delta_{\text {rough }}$ a roughness parameter. If the gap $h_{i j}^{\perp}$ is negative (and $\delta^{\perp}$ nonzero), the lubrication is set equal to 0 . We have equally accounted for the weaker shear mode,

$$
\mathbf{f}_{\text {lub }, t}^{i j}=\left[-\frac{1}{2} \pi \eta_{f} \ln \left(\frac{\kappa_{i j}^{-1}}{2\left(h_{i j}^{\perp}+\delta_{\text {rough }}\right)}\right)\left(\mathbf{V}_{i}-\mathbf{V}_{j}\right) \cdot \mathbf{t}_{i j}\right] \mathbf{t}_{i j},
$$

where $\mathbf{t}_{i j}$ is the tangential vector at the closest contact points. We chose to have the roughness parameter equal to $\delta_{\text {rough }} / \sqrt{\langle a b\rangle}=0.025$.

Figure 8 shows that including lubrication forces does not diminish the directional shear jamming. These simulations have been run with Newtonian dynamics but should be considered as overdamped, as the Stokes number $\mathrm{St}=$ $\frac{\rho \dot{\gamma} \sqrt{a b}}{\eta_{f}} \ll 1$, where $\rho$ is the mass density of particles. 


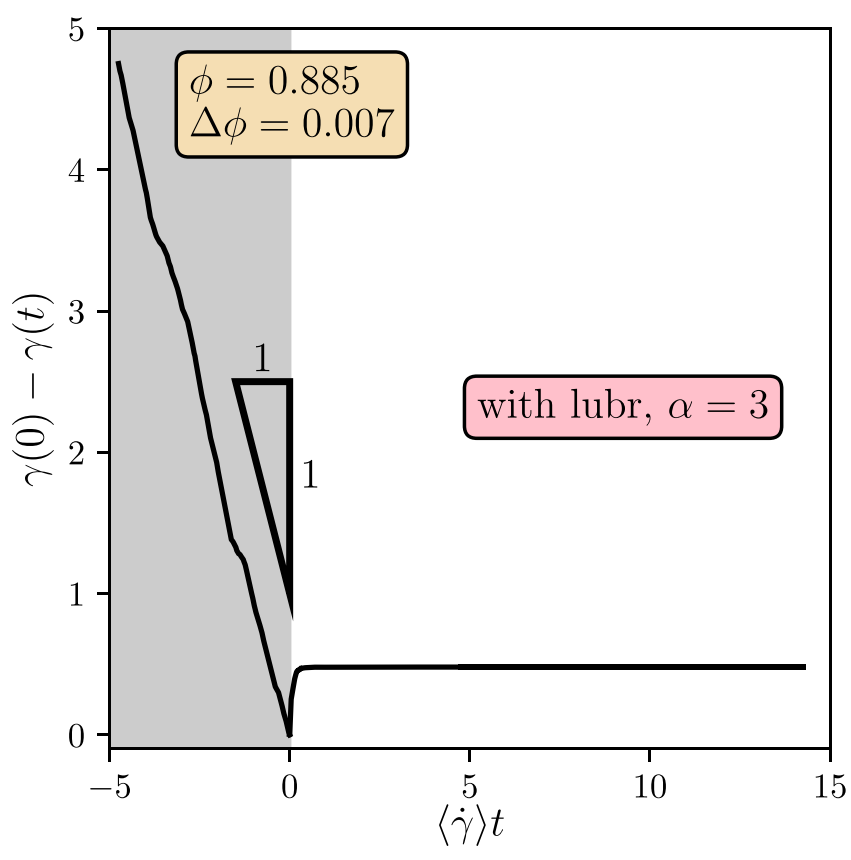

FIG. 8. As Fig. 1(d) in the text, with lubrication forces included $\left(\mathrm{St}=2.5 \times 10^{-3}\right)$.

\section{APPENDIX C: EFFECT OF WALL ROUGHNESS}

In the text, we altered the wall particles' shapes. In order to explore whether directional shear jamming is caused by the boundary, we carry out two extra simulations. The first is by altering only the wall particles' shapes, keeping the flowing particles as disks. The other test is doing the reverse, i.e., keeping the wall particles as disks but changing the flowing particles' shapes. As shown in Fig. 9, the wall roughness does affect the dynamics and, hence, does not alter the conclusion reached in the text. Therefore, we can conclude that directional shear jamming is not a boundary-driven effect.

\section{APPENDIX D: FINITE-SIZE EFFECTS}

In order to investigate whether directional shear jamming is caused by the finite separation between the walls or the finite (periodic) width of the simulation box, we carry out
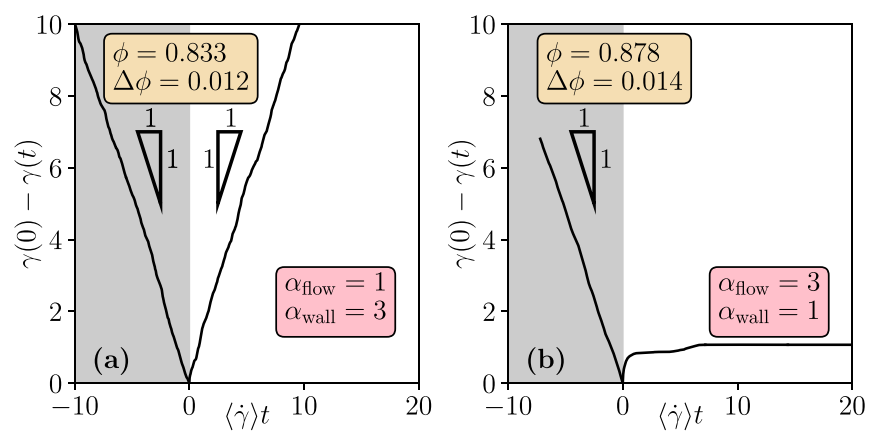

FIG. 9. Strain $\gamma$ as a function of the rescaled time $\langle\dot{\gamma}\rangle t$. (a) Ellipse walls with flowing disks and (b) disk walls with flowing ellipses.
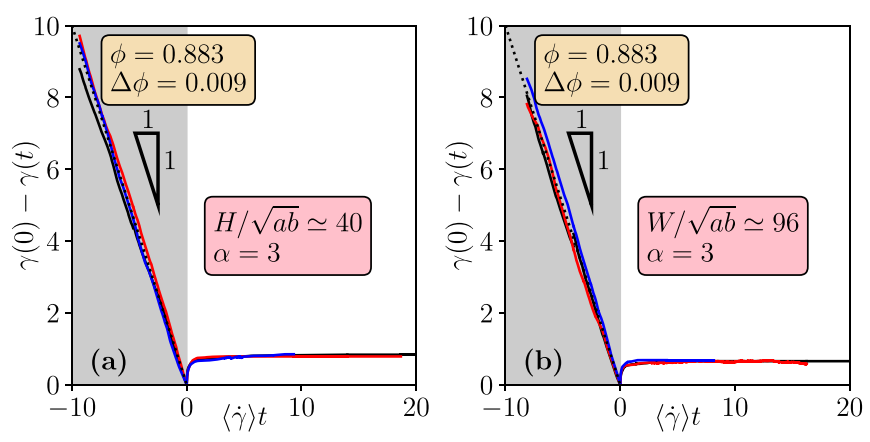

FIG. 10. As Fig. 1(d) in the text, but with (a) twice the wall separation or (b) twice the (periodic) box width.

additional simulations with double separation between the walls and doubling of the width of the simulation box, both corresponding to roughly 1800 particles rather than 900. As shown in Fig. 10 and by comparison with Fig. 1(d) in the text, directional shear jamming persists even for larger systems.

\section{APPENDIX E: RESULTS FOR A LARGER SPRING CONSTANT}

Figure 11 shows the results for a 10 times larger spring constant, $k /\langle P\rangle \sim 10^{4}$. While the viscous number $J \sim 10^{-3}$ is comparable to that in Fig. 1(c) in the text, both $\sigma$ and $\dot{\gamma}$ are one order of magnitude smaller than in the text. Importantly, we see that also the stiffer particles (compared to the shear pressure) display directional shear-jamming behavior.

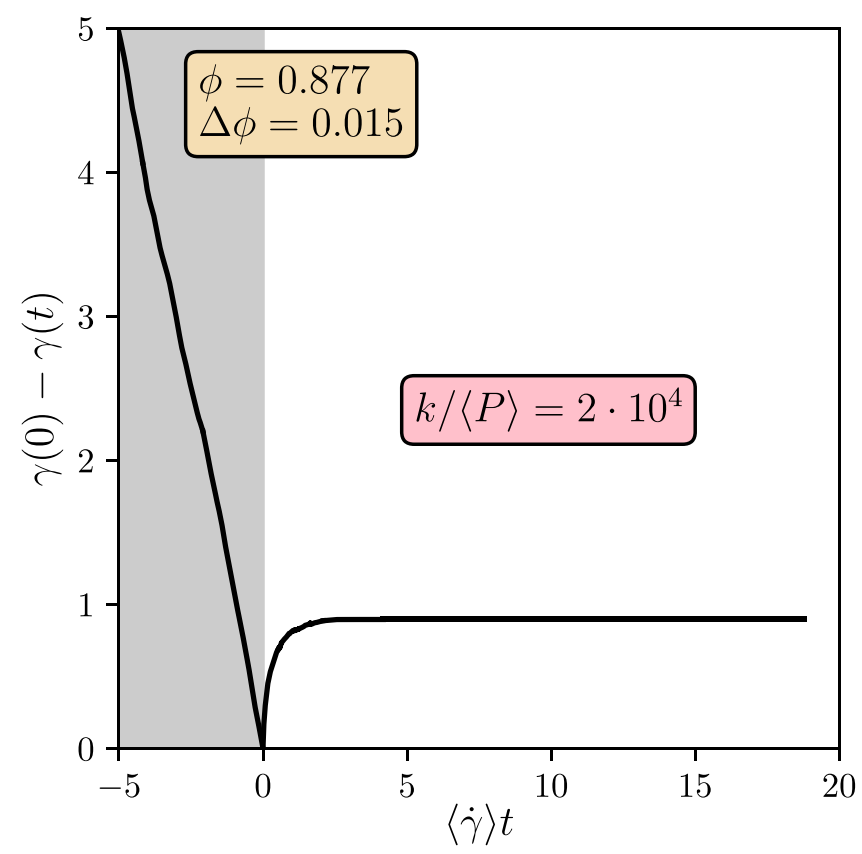

FIG. 11. As Fig. 1(c) in the text, but with a 10 times larger spring constant. 
[1] B. Andreotti, Y. Forterre, and O. Pouliquen, Granular Media: Between Fluid and Solid (Cambridge University Press, Cambridge, UK, 2013).

[2] E. Lerner, G. Düring, and M. Wyart, A unified framework for non-Brownian suspension flows and soft amorphous solids, Proc. Natl. Acad. Sci. USA 109, 4798 (2012).

[3] P. Olsson, Dimensionality and Viscosity Exponent in ShearDriven Jamming, Phys. Rev. Lett. 122, 108003 (2019).

[4] A. Baule, F. Morone, H. J. Herrmann, and H. A. Makse, Edwards statistical mechanics for jammed granular matter, Rev. Mod. Phys. 90, 015006 (2018).

[5] M. M. Denn and J. F. Morris, Rheology of non-Brownian suspensions, Annu. Rev. Chem. Biomol. Eng. 5, 203 (2014).

[6] E. Guazzelli and O. Pouliquen, Rheology of dense granular suspensions, J. Fluid Mech. 852, P1 (2018).

[7] S. M. Fielding, Shear banding in soft glassy materials, Rep. Prog. Phys. 77, 102601 (2014).

[8] R. Seto, R. Mari, J. F. Morris, and M. M. Denn, Discontinuous Shear Thickening of Frictional Hard-Sphere Suspensions, Phys. Rev. Lett. 111, 218301 (2013).

[9] M. Wyart and M. Cates, Discontinuous Shear Thickening without Inertia in Dense Non-Brownian Suspensions, Phys. Rev. Lett. 112, 098302 (2014).

[10] J. Dong and M. Trulsson, Analog of discontinuous shear thickening flows under confining pressure, Phys. Rev. Fluids 2, 081301(R) (2017).

[11] J. Dong and M. Trulsson, Unifying viscous and inertial regimes of discontinuous shear thickening suspensions, J. Rheol. 64, 255 (2020).

[12] D. J. Pine, J. P. Gollub, J. F. Brady, and A. M. Leshansky, Chaos and threshold for irreversibility in sheared suspensions, Nature 438, 997 (2005).

[13] L. Corté, P. M. Chaikin, J. P. Gollub, and D. J. Pine, Random organization in periodically driven systems, Nat. Phys. 4, 420 (2008).

[14] G. I. Taylor, Kinematic reversibility, in Multi-Media Fluid Mechanics CD-ROM, edited by G. M. Homsy (Cambridge University Press, Cambridge, UK, 2000).

[15] M. Souzy, P. Pham, and B. Metzger, Taylor's experiment in a periodically sheared particulate suspension. Phys. Rev. Fluids 1, 042001(R) (2016).

[16] P. Das, H. A. Vinutha, and S. Sastry, Unified phase diagram of reversible-irreversible, jamming, and yielding transitions in cyclically sheared soft-sphere packings, Proc. Natl. Acad. Sci. USA 117, 10203 (2020).

[17] N. Y. C. Lin, B. M. Guy, M. Hermes, C. Ness, J. Sun, W. C. K. Poon, and I. Cohen, Hydrodynamic and Contact Contributions to Continuous Shear Thickening in Colloidal Suspensions, Phys. Rev. Lett. 115, 228304 (2015).

[18] C. Ness, R. Mari, and M. E. Cates, Shaken and stirred: Random organization reduces viscosity and dissipation in granular suspensions, Sci. Adv. 4, eaar3296 (2018).

[19] J. Dong and M. Trulsson, Transition from steady shear to oscillatory shear rheology of dense suspensions, Phys. Rev. E 102, 052605 (2020).
[20] R. Seto, A. Singh, B. Chakraborty, M. M. Denn, and J. F. Morris, Shear jamming and fragility in dense suspensions, Granul. Matter 21, 82 (2019).

[21] D. Bi, J. Zhang, B. Chakraborty, and R. B. Behringer, Jamming by shear, Nature 480, 355 (2011).

[22] Y. Zhao, J. Barés, H. Zheng, J. E. S. Socolar, and B. Behringer, Shear-Jammed, Fragile, and Steady States in Homogeneously Strained Granular Materials, Phys. Rev. Lett. 123, 158001 (2019).

[23] M. Trulsson, Rheology and shear jamming of frictional ellipses, J. Fluid Mech. 849, 718 (2018).

[24] A. Chwang and T. Wu, Hydromechanics of low-Reynoldsnumber flow. Part 2. Singularity method for Stokes flows, J. Fluid Mech. 67, 787 (1975).

[25] L. Berthier and G. Biroli, Glasses and aging, a statistical mechanics perspective on, in Encyclopedia of Complexity and Systems Science, edited by R. Meyers (Springer, New York, 2009).

[26] T. Börzsönyi, B. Szabó, S. Wegner, K. Harth, J. Török, E. Somfai, T. Bien, and R. Stannarius, Shear-induced alignment and dynamics of elongated granular particles, Phys. Rev. E 86, 051304 (2012).

[27] B. Nadler, F. Guillard, and I. Einav, Kinematic Model of Transient Shape-Induced Anisotropy in Dense Granular Flow, Phys. Rev. Lett. 120, 198003 (2018).

[28] T. A. Marschall, D. Van Hoesen, and S. Teitel, Shear-driven flow of athermal, frictionless, spherocylinder suspensions in two dimensions: Particle rotations and orientational ordering, Phys. Rev. E 101, 032901 (2020).

[29] Z. Zheng, R. Ni, F. Wang, M. Dijkstra, Y. Wang, and Y. Han, Structural signatures of dynamic heterogeneities in monolayers of colloidal ellipsoids, Nat. Commun. 5, 3829 (2014).

[30] A. F. de Coulomb, M. Bouzid, P. Claudin, E. Clément, and B. Andreotti, Rheology of granular flows across the transition from soft to rigid particles, Phys. Rev. Fluids 2, 102301(R) (2017).

[31] T. Bertrand, R. P. Behringer, B. Chakraborty, C. S. O'Hern, and M. D. Shattuck, Protocol dependence of the jamming transition, Phys. Rev. E 93, 012901 (2016).

[32] D. B. Nagy, P. Claudin, T. Börzsönyi, and E. Somfai, Rheology of dense granular flows for elongated particles, Phys. Rev. E 96, 062903 (2017).

[33] F. Boyer, E. Guazzelli, and O. Pouliquen, Unifying Suspension and Granular Rheology, Phys. Rev. Lett. 107, 188301 (2011).

[34] M. Trulsson, B. Andreotti, and P. Claudin, Transition from the Viscous to Inertial Regime in Dense Suspension, Phys. Rev. Lett. 109, 118305 (2012).

[35] L. Amarsid, J.-Y. Delenne, P. Mutabaruka, Y. Monerie, F. Perales, and F. Radjai, Viscoinertial regime of immersed granular flows, Phys. Rev. E 96, 012901 (2017).

[36] J. Dong and M. Trulsson, Oscillatory shear flows of dense suspensions at imposed pressure: Rheology and micro-structure, arXiv:2011.13215. 